\title{
Quality assurance of thermodynamic data
}

\author{
Stefan Lis • Günther Meinrath
}

Published online: 19 March 2011

(C) Springer-Verlag 2011

To promote metrology in chemistry, ACQUAL has invited a Topical Issue under the header "Quality Assurance in Chemical Thermodynamics". Chemical thermodynamics is an important field of experimental chemistry. Its results are widely used even outside the chemical field. Data collections of formation quotients of chemical species in aqueous solutions are compiled with the intention to simulate the behavior of ground water constituents; activity coefficients are determined in order to predict the solubility of green house gases in ocean seawater; the thermodynamic properties of newly prepared compounds are tested for their interaction in industrial processes.

The initial intention has been to learn how current workers in the field assess the quality of the data published under their name. The number of possible contributors for such an issue is, however, limited. Next to the often protracted experimental work, complex numerical (e.g., chemometric) methods need to be found to obtain an appropriate model or to decide between alternative appropriate models for the interpretation of the observed data. Only a limited number of workers in chemical thermodynamics are at least willing to deal with metrological issues. Therefore, the title of this topical issue has been decided to be "quality assurance of thermodynamic data," not "metrology of thermodynamic data."

\footnotetext{
S. Lis $(\square)$

Department of Rare Earths, Faculty of Chemistry,

Adam Mickiewicz University, Grunwaldzka 6, 60-780 Poznań, Poland

e-mail: blis@amu.edu.pl

G. Meinrath

RER Consultants Passau, 94036 Passau, Germany
}

There are multiple possible reasons. The complexity of a thermodynamic study, e.g., of metal ligand interaction in aqueous solution, is but one reason. The application of sophisticated numerical models in the analysis of experimental data confronts the researchers with multiple possible interpretations often without clear criteria to make a decision. Targeting the behavior of chemicals in the environment may raise the problem to define the measurand. Naturally occurring organic materials (NOM), for instance, form important reactants in the environment, however, without allowing a simple separation into chemically identifiable compounds with unambiguous molar masses. As long as there is no real demand, this situation will change with an only low momentum.

"Chemistry is a study of complicated systems and the complexity cannot be understood at an arbitrary level of accuracy. (...) Thus any measured data has noise, uncertainties and variability's, non-linearity's and unrecognized features (model-deficiencies). And mainstream chemistry has still great difficulties in recognising these basic facts" [1]. These words might be from an ACQUAL editorial but in fact are stated 15 years ago by one of the founding fathers of chemometrics. Statistical and numerical techniques are among those methods also applied by thermodynamicians to assess variability in data. However, the reader should understand that incorporation of metrological concepts into chemical thermodynamics is a process at its beginnings-and not yet a success story. While the complete measurement uncertainty budget is a topic, the important concept of traceability, for instance, is not discussed at all.

This issue, nevertheless, consists an interesting fixing point of the current state-of-the-art in metrology in chemistry. It is probably easy to understand that a reviewer from the field of thermodynamics sees little interest in a complex 
numerical method just deriving an uncertainty limit. Thus, it is difficult to place a related manuscript in the thermodynamics literature. The principal motivation of this issue has been to bring different fields together to further mutual understanding. Mutual understanding requires openness to alternative approaches. Among the various aspects, this issue has highlighted that more openness on all sides seems to be a prime necessity for gaining acceptance for metrology in chemistry. Such an ambition for acceptance is especially needed in such fields where consideration of metrological aspects cannot be enforced by regulatory pressure (e.g., by a standard like ISO 17025). This issue therefore may contribute to a mutual learning process by confronting the available concepts with the existing needs.

Stefan Lis

Günther Meinrath

Guest Editors, Topical Issue "Quality Assurance of Thermodynamic Data"

\section{Reference}

1. Wold S (1995) Chemometrics Intel Lab Syst 30:109-115 